\title{
Total Parenteral Nutrition Catheter Sepsis: Impact of the Team Approach*
}

\author{
Walter C. Faubion, M.S., R.N., John R. Wesley, M.D., Nabil Khalidi, Pharm.D., and \\ Joseph Silva, M.D.
}

From the Parenteral and Enteral Nutrition Team, the Departments and Schools of Nursing, Pharmacy, Medicine, and Surgery, University of Michigan Medical Center, Ann Arbor, Michigan

\begin{abstract}
For a period of 32 months from the inception of the Parenteral and Enteral Nutrition (PEN) Team at the University of Michigan, the infection rate (IR) related to central venous catheters (CVCs) for total parenteral nutrition (TPN) was prospectively evaluated. Six hundred twenty-two catheters were placed in 377 patients for a total of 9,200 patient days. The length of CVC stay ranged from 2 to 108 days with a mean of 14.5. CVC longevity was longer on units where certification of nurses by the PEN team was mandatory (mean 20.4 days, IR $3.5 \%$ ) vs comparable units which utilized informal instruction (mean 14.4 days, IR 3.5\%). Twenty-two catheters
\end{abstract}

led to patient sepsis for a rate of $3.5 \%$, or 2.39 CVC-related infections per 1000 patient days. The preteam infection rate was $24.0 \%$, as determined by a 6 -month prospective study. Infection rates for CVCs used for TPN only and those used for parenteral nutrition (PN) plus blood products, antibiotics, and CVP measurements were identical, 3.5\%. The team approach can effectively increase PN catheter longevity and reduce infection rates. Conservative cost accounting of best and worst case scenarios determined a range of $\$ 3,700$ to $\$ 8,900$ per episode of catheter sepsis. (Journal of Parenteral and Enteral Nutrition 10: $642-645,1986$ )
The number of nutrition support teams in hospitals providing parenteral nutrition has grown over the last decade. Teams were started with the goal of decreasing the rate of catheter-related sepsis and metabolic complications. ${ }^{1-5}$ Although a number of reports have appeared documenting low catheter sepsis rates in institutions with nutrition support teams, ${ }^{2,6-8}$ few institutions had mechanisms set up to prospectively compare preteam catheter infection rates with postteam rates.

Recognizing the morbidity, expense, and potential mortality of catheter-related sepsis, the Division of Infectious Disease at the University of Michigan Medical Center in 1974 documented a $24.0 \%$ incidence of central venous catheter sepsis in 162 patients with 179 catheter insertions (catheters infected/catheters at risk) during a 6 -month period prior to the inception of our nutritional support team. ${ }^{9}$ With the knowledge of an unacceptably high preteam infection rate, the newly formed Parenteral and Enteral Nutrition (PEN) Team set up a prospective study to evaluate the effectiveness of the team in the area of catheter-related sepsis.

The present report documents a reduction of $85 \%$, or 20.5 percentage points, in catheter sepsis over the first 32 months from the inception of the team and discusses the cost-effective impact of the team approach to parenteral nutrition. The reduction occurred within the first 6 months and was maintained for the duration of the study.

Received for publication. October 24, 1985

Accepted for publication, March 24, 1986.

Reprint requests: Dr. John R. Weslev. Pediatric Surgerv, Fiol6 Mott Childrens Hospital. 1405 E. Ann Street/Box 0245. Ann Arbor, MI 48109 .

* Presented at the Sixth Clinical Congress, A.S.P.E.N., San Francisco, CA, February 3-6, 1982.

\section{METHODS}

The methods for the preteam study have been previously outlined by J. Silva. ${ }^{9}$

During a 32-month period all central venous catheters (CVCs) used for parenteral nutrition $(\mathrm{PN})$ were monitored. A strict aseptic protocol for CVC placement and dressing changes were supervised by members of the PEN team. ${ }^{10}$ All catheters were placed by medical or surgical house officers, or staff physicians.

The placement was done in a variety of locations including operating rooms, intensive care units, treatment rooms, and the patient's bedside. All persons present during the procedure were masked. Persons placing the catheter also wore sterile gloves. Some physicians chose to wear gowns and operating room (OR) caps as well, although this was not mandatory. The patient was generally not masked as a sterile drape was placed over the neck and face.

The types of catheters placed included: Intracath (Deseret Company, Sandy, UT), central venous pressure ports of Swan Ganz thermodilution catheters (Edwards Labs, Puerto Rico), and the side port of the Cordis catheter (Cordis Corp., Miami, FL). CVCs were placed in the jugular as well as subclavian veins. The site chosen depended on available venous access and physician preference.

Sterile dressing changes were done every $48 \mathrm{hr}$ using a dressing change kit designed by the PEN team and supplied by Concord (Keenan, NH).

Masks were worn by the patient and nurse as well as any other person in the room. The area of insertion was first inspected for drainage or swelling and then cleansed using a clean-to-dirty technique with three alcohol-acetone swabsticks, followed by three povidone iodine swab- 
sticks. Povidone iodine ointment was then placed on the insertion and suture sites. The sterile luer lock extension tubing was changed and the entire hub-extension tube junction as well as the catheter was covered by sterile gauze. A water repellent tape was applied rendering the dressing occlusive.

Nurses on all of the pediatric units serving PN patients and nurses on one adult surgical floor were selected for training and certification in the techniques of PN dressing changes. The training program was conducted by the PEN team nurse clinicians in an effort to reduce catheter sepsis. Hospital policy directed that all CVCs for PN were to be used for PN only. Exceptions were made for selected patients with limited venous access.

CVC information was collected on a data collection form and analyzed by computer. Presumptive sepsis was defined as CVC related if the catheter tip culture was positive, if there was a positive blood culture with no other source of infection, or if defervescence followed CVC removal. All CVCs were included even if there were other sites of documented infection. All cultures yielding organisms were reviewed and confirmed by the Division of Infectious Disease.

\section{RESULTS}

Three hundred seventy-seven (377) patients had 622 central venous catheters placed for parenteral nutrition during the study period, for a total of 9200 patient days. The length of catheter insertion varied from 2 to 108 days with a mean of 14.5 days. Twenty-two of the catheters became infected. The culture results are found in Table I. The overall infection rate was $3.5 \%$ or 2.39 infected catheters per 1000 patient days.

The infection rate for multiple use catheters $(3.5 \%)$ was exactly the same as the rate for catheters used only for parenteral nutrition (Table II). However, the threshold for removing multiple use catheters was lower than for catheters used for PN alone because of the suspicion on the part of the physician that these catheters were more likely to become septic (Table III).

There was no difference in catheter infection between the areas of the hospital where we required certification for nurses performing catheter dressing change and those where certification was not required, with identical infection rates of $3.5 \%$. The mean length of catheter stay, however, was 6 days longer in the group whose catheter dressing changes were performed by certified personnel, 20.4 us 14.4 days.

As documented in Table IV patients who had more than one catheter were more likely to have a second or third catheter infected than the first catheter. The catheter infection rate was $2.7 \%$ for patients who developed infection in their first catheter, while infection rates for subsequent catheters varied from 0 to $9 \%$ with a cumulative infection rate of $4.9 \%$ for catheters two through seven. Only two patients out of the group of 20 had a second episode of catheter sepsis.

Using a test for trends, no significant difference was found between infection rates for short term CVCs lless than 1 week) and long term CVCs (greater than I week). $p<0.16$ (Table V).
TABLE I

Organisms cultured from the catheter tip and/or from blood draun through the catheter. In 1974 the majority of the colonized catheters uere Candida (12), coagulase negative staphvlococcus (8), and Klebsiella sp. (7).

Infected catheter cultures

10 coagulase $(-)$ staphylococcus

10 coagulase $(+)$ staphylococcus

1 Candida albicans

1 group D enterococcus, Serratia sp.

TABLE II

Infection rate in catheters used for parenteral nutrition only vs catheters which had multiple uses including parenteral nutrition

\begin{tabular}{lccc} 
& $\begin{array}{c}\text { CVCs } \\
\text { employed } \\
\text { for PN } \\
\text { only }\end{array}$ & $\begin{array}{c}\text { Multiuse } \\
\text { CVCs }\end{array}$ & Total \\
\hline Infected CVCs & 18 & 4 & 22 \\
Total CVCs & 509 & 113 & 622 \\
Rate (\%) & 3.5 & 3.5 & 3.5 \\
\hline
\end{tabular}

TABLE III

Catheters removed for suspected sepsis. PN only compared with multiple use catheters

\begin{tabular}{ccc}
$\begin{array}{c}\text { CVCs } \\
\text { employed } \\
\text { for PN } \\
\text { only }\end{array}$ & $\begin{array}{c}\text { Multiunt } \\
\text { (Vi) }\end{array}$ & Total \\
129 & 37 & 166 \\
509 & 113 & 622 \\
25.3 & 32.7 & 26.7 \\
\hline
\end{tabular}

TABLE IV

Comparison of infection rates in 20 patitnts wh infection in the first or subsequent catheters

\begin{tabular}{lccccccc}
\hline & \multicolumn{7}{c}{ Catheter } \\
\cline { 2 - 8 } & 1st & 2nd & 3rd & 4th & 5th & 6th & 7th + \\
\hline No. CVC infected & 10 & 8 & 3 & 0 & 0 & 1 & 0 \\
No. CVC at risk & 377 & 123 & 58 & 30 & 16 & 11 & 7 \\
Rate $(c)$ & 2.7 & 6.3 & 5.0 & & & 9.1 & \\
\hline
\end{tabular}

TABI,E V

Comparison of infection ratex and the longerity of the indurlling cathe'ter

\begin{tabular}{lcccccc}
\hline & \multicolumn{7}{c}{ Days } \\
\cline { 2 - 7 } & $1-7$ & $8-14$ & $15-21$ & $22-28$ & $29+$ & Total \\
\hline No. infect & 3 & 6 & 4 & 5 & 4 & 22 \\
No. CVCs & 175 & 218 & 97 & 68 & 64 & 622 \\
Rate (\%) & 1.58 & 2.75 & 4.12 & 7.35 & 6.25 & 3.54 \\
\hline
\end{tabular}

\section{DISCUSSION}

Since the inception of the PEN team. our CVC sepsis rate of $3.5 \%$ has been well below the ${ }^{\circ} c$ average rate for institutions with established parenteral nutrition guidelines as reported by the Center for Disease Control." Our rate is similar to rates reported by other instiutions where intensive etforts have been $u$ : "n to decrease metabolic and septic complicati ciated with PN. ${ }^{12-14}$ This rate is a marked improsement wer the $24 \%$ (12 of 501 intection rate for $P N$ catheters that the Division of Intectious Disease had documented earlier. ${ }^{9}$ 
The earlier report was a prospective 6 -month study which investigated all CVCs placed in the institution and related positive blood cultures and tip cultures to the total number of catheters at risk by usage groups. At our hospital, PN catheters are inserted by medical and surgical residents and dressing changes are performed largely by staff nurses according to an established PEN team protocol. It is frequently difficult to compare infection rates between institutions since not all institutions have the same criteria for including catheters into PNrelated sepsis statistics or the same definition of catheter sepsis.

Catheters used for PN have traditionally been reserved for that purpose alone, but perhaps that restriction is not always necessary. Although the infection rate in multiple-use catheters was not significantly different from that in catheters used for $\mathrm{PN}$ alone, we still recommend that PN catheters be used for PN alone to insure timely and uninterrupted flow. Multiple use should be reserved for those patients where no other vascular access is available.

An unexpected finding of our study was that of no difference in infection rates for the group of patients cared for by nurses certified in CVC dressing change techniques $v s$ uncertified nurses. All nurses had exposure to the CVC dressing change procedure and hospital policy during orientation to the units. Certified nurses were shown a dressing change on the patient and then were expected to perform a dressing change at a later date with a PEN team nurse in attendance. This demonstration may have provided more practical information on maintaining the CVCs. The patients cared for by the certified group had an average length of stay that was 6 days longer than the noncertified group. They also had fewer mechanical problems leading to removal of catheters. The certification group consisted of those nurses taking care of pediatric patients, and those working on the surgical ward with the greatest acuity and largest PN population. One might have expected this group to have had a shorter length of CVC longevity and a higher sepsis rate. It appears that strict adherance to protocol and close monitoring can lead to a decreased sepsis rate and increased catheter longevity.

Second and third CVCs were placed in patients for reasons that included suspected sepsis, clotting, mechanical failure, accidental removal, and desire for a new line. One patient had 15 catheters placed during his hospitalization. The new CVCs were generally placed on the opposite side or in a different venous access location within 24 to $48 \mathrm{hr}$. There is a possibility that those patients who received more than one CVC were more seriously ill and were, consequently, at greater risk for infection overall. Based on this information and the lack of association between sepsis and CVC longevity it would appear prudent to make every effort to maintain the original catheter. These data are similar to what others have reported. ${ }^{6.12,15}$ By contrast, in the 1974 study prior to the PEN team, the colonization rate was 17 of 109 if left in place 0 to 7 days and 23 of 30 if left in place 8 to 30 days.

Nutritional support teams have helped to make parenteral nutrition safe and less costly for the patient and
TABLE VI

Estimated cost of catheter-related sepsis using best and worst case scenarios

\begin{tabular}{lc}
\hline Best case: Extended stay 7 days, sensitive organism, Nafcillin \\
( 1 g, every $6 \mathrm{hr}$ ) & \\
Ward room $\$ 378 /$ day ( $\times 7$ days) & 2646 \\
Work up cultures & 212 \\
Bacteriology & 378 \\
Antibiotics & 98 \\
IV fluids and tubing & 83 \\
Repeat cultures $\times 2$ & 40 \\
Repeat CBC & $\$ 3699$ per patient
\end{tabular}

Worst case: Extended stay by 10 days, resistant organism requiring a private room and isolation, Vancomycin $(500 \mathrm{mg}$, every $6 \mathrm{hr}$ )

Room $\$ 608 \times 10$ days $\$ 6080$

Work up cultures $r$

Bacteriology $\quad 212$

Antibiotics 1296

IV fluids and tubing $\quad 98$

Repeat cultures $\times 2 \quad 83$

Repeat CBC

Isolation equipment

40

840

$\overline{\$ 8891}$ per patient

TABLE VII

Monthly expenses of the PEN Team

$\begin{array}{lr}\text { Nurses (3) } & \$ 7,600 \\ \text { Pharmacists (2) } & 5,000 \\ \text { Nutritionist (1) } & 2,400 \\ \text { Secretary (1) } & 1,250 \\ \text { Students, supplies, misc. } & 2,950 \\ \quad \text { Total } & \$ 19,100\end{array}$

the institution. Although metabolic complications are reduced in hospitals with nutritional support teams, it is difficult to determine the monitary value of such an approach. Sepsis, on the other hand, is clearly very costly. In an attempt to estimate these cost savings, we have compared our preteam and current rates of sepsis. Using a best case and worst case scenario, cost ranges for sepsis were developed (Table VI).

Based on the number of catheters in our study, the number of septic catheters predicted if the team had not been in place is 149 . Therefore, assuming a $24.0 \%$ infection rate without the team approach, the calculated cost over the 32 months would have ranged from $\$ 551,151$ (best case) to $\$ 1,324,759$ (worst case). However, with the PEN team's intervention (3.5\% infection rate) the cost ranged from $\$ 81,378$ to $\$ 195,602$ over the same 32 months. The savings then ranged from $\$ 14,680$ to $\$ 35,286$ per month, which compares favorably to the PEN team's total operating budget of $\$ 19,100$ per month in 1985 dollars (Table VII). This saving assumes increasing significance in light of the prospective payment reimbursement programs.

\section{CONCLUSION}

The team approach to PN catheter care can effectively increase PN catheter longevity and reduce infection rates. Parenteral nutrition lines can be employed for multiple purposes without fear of increased sepsis if used carefully. A parenteral nutrition team is almost certainly 
cost effective when comparing the cost of sepsis to the team's operating budget.

\section{ACKNOWLEDGMENTS}

The authors express their thanks to Wendy L. Baker, R.N., Beth A. Perlmutter, R.N., and Alan K. Leibowitz for technical assistance, and to Josephine Bender, R.N. for the cost analysis.

\section{REFERENCES}

1. Maki DG: Sepsis arising from extrinsic contamination of the infusion and measures for control. IN Microbiologic Hazards of Intravenous Therapy, Phillips I, Meers PD D'Arch PF (eds). MTP Press Ltd, Lancaster, England, 1977, pp 99-144

2. Allen JR: The incidence of nosocomial infection in patients receiving total parenteral nutrition. IN Advances in Parenteral Nutrition, Proceedings of an International Symposium, Bermuda, May 16-19, 1977, Johnston IDA (ed). MTP Press Ltd, Lancaster, England, 1977, pp 339-377

3. Cury CR, Quie PG: Fungal septicemia in patients receiving parenteral alimentation. N Engl $J$ Med 285:1221-1224, 1971

4. Ashcraft KW, Leape LL: Candida sepsis complicating parenteral feeding. JAMA 212:454-456, 1970
5. Dudrick S.J. Groff DB. Wilmore DW: Long term venous catheterization in infants. Surg Gynecol Obstet 129:805-808, 1969

6. Sanders RA, Sheldon GF: Septic complications of total parenteral nutrition. Am J Surg 132:214-2:20. 1976

7. Nehme AE: Nutritional support of the hospitalized patient: The team concept. JA.MA 243:1906-1908, 1980

8. Padberg FT, Ruggiero J, Blackburn GL. Bistrian BR: Central venous catheterization for parenteral nutrition. Ann Surg 193:264270,1981

9. Silva J, Prager RL. Harkema J: Infections of central venous catheters. Clin Res 24:546A. 1976

10. Wesley JR, Khalidi N, Faubion WC, et al: The Lniversity of Michigan Hospitals Parenteral and Enteral Nutrition Manual, Abbott Laboratories, June, 1984

11. Guidelines for Infectious Control in Hyperalimentation Therapy. National Nosocomial Infections Study Quarterly Report, 22-25, May, 1972

12. Ryan JA, Abel RM, Abbott WM, et al: Catheter complications in total parenteral nutrition. A prospective study of 200 consecutive patients. N Engl J Med 290:757-761, 1974

13. Sanderson I, Deitel M: Intravenous hyperalimentation without sepsis. Surg Gynecol Obstet 136:577-585, 1973

14. Brown RS, Grenkoski J: Total Parenteral Nutrition: A safe procedure in the small community hospital? Crit Care Med 5:241-244, 1977

15. Bernard RW, Stahl WM, Chase RM, Jr.: Subclavian vein catheterizations: A prospective study. II. Infectious complications. Ann Surg 173:191-200, 1971 